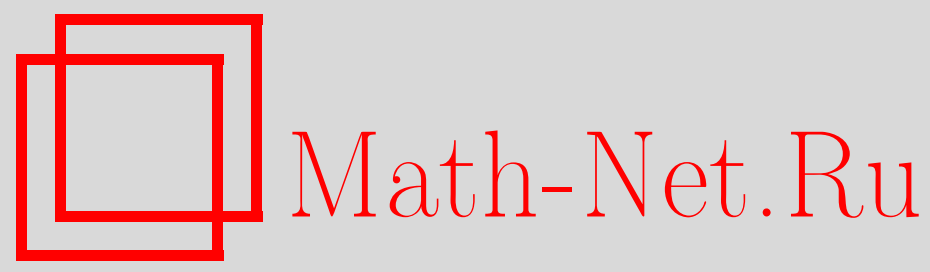

М. О. Корпусов, А. Г. Свешников, О "разрушении" решений нелинейных волновых уравнений типа Соболева с кубическими источниками, Матем. заметки, 2005, том 78, выпуск 4, 559-578

DOI: https://doi.org/10.4213/mzm2614

Использование Общероссийского математического портала Math-Net.Ru подразумевает, что вы прочитали и согласны с пользовательским соглашением http://www . mathnet.ru/rus/agreement

Параметры загрузки:

IP : 54.197 .130 .99

26 апреля 2023 г., 18:32:57

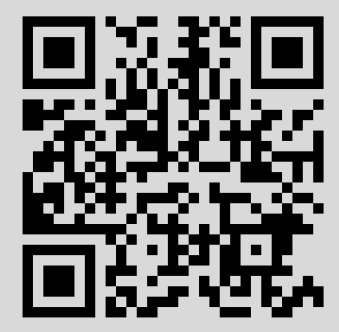




\title{
О “РАЗРУШЕНИИ" РЕШЕНИЙ НЕЛИНЕЙНЫХ ВОЛНОВЫХ УРАВНЕНИЙ ТИПА СОБОЛЕВА \\ С КУБИЧЕСКИМИ ИСТОЧНИКАМИ
}

\author{
М. О. Корпусов, А.Г. Свешников
}

\begin{abstract}
Рассматриваются модельные трехмерные волновые нелинейные уравнения типа Соболева с кубическими источниками. В первую очередь модельные трехмерные уравнения типа Бенджамена-Бона-Махони и Розенау с модельными кубическими источниками. Также рассмотрено существенно трехмерное нелинейное уравнение спиновых волн с кубическим источником. Для данных уравнений рассматривается первая начально-краевая задача в ограниченной области с гладкой границей. Доказана локальная разрешимость в сильном обобшенном смысле, а для уравнения типа Бенджамена-Бона-Махони с источником доказана однозначная разрешимость "ослабленного" решения. Получены достаточные условия "разрушения" решений рассмотренных задач. Эти условия имеют смысл "болшшой" величины начального возмущения по нормам некоторых банаховых пространств. Наконец, для уравнения типа Бенджамена-Бона-Махони доказано "опрокидывание" "ослабленного" решения за конечное время.
\end{abstract}

Библиография: 28 названий.

1. Введение. Постановка задачи. Целью настоящего исследования является получение достаточных условий разрушения решений следующих первых начально-краевых задач:

$$
\begin{aligned}
& \frac{\partial}{\partial t}(\Delta u-u)+u \frac{\partial u}{\partial x_{1}}+u^{3}=0,\left.\quad u\right|_{\partial \Omega}=0, \quad u(x, 0)=u_{0}(x) ; \\
& \frac{\partial}{\partial t}\left(-\Delta^{2} u+\Delta u\right)+u \frac{\partial u}{\partial x_{1}}-\operatorname{div}\left(|\nabla u|^{2} \nabla u\right)=0,\left.\quad u\right|_{\partial \Omega}=\left.\frac{\partial u}{\partial \mathbf{n}}\right|_{\partial \Omega}=0, \quad u(x, 0)=u_{0}(x) ; \\
& \frac{\partial}{\partial t}\left(-\Delta^{2} u+\Delta u\right)-\operatorname{div}\left(|\nabla u|^{2} \nabla u\right)+\beta_{1} \frac{\partial}{\partial x_{1}}\left(\frac{\partial u}{\partial x_{2}} \frac{\partial u}{\partial x_{3}}\right)+\beta_{2} \frac{\partial}{\partial x_{2}}\left(\frac{\partial u}{\partial x_{3}} \frac{\partial u}{\partial x_{1}}\right) \\
& \quad+\beta_{3} \frac{\partial}{\partial x_{3}}\left(\frac{\partial u}{\partial x_{1}} \frac{\partial u}{\partial x_{2}}\right)=0, \quad \beta_{1}+\beta_{2}+\beta_{3}=0,\left.\quad u\right|_{\partial \Omega}=\left.\frac{\partial u}{\partial \mathbf{n}}\right|_{\partial \Omega}=0, u(x, 0)=u_{0}(x),
\end{aligned}
$$

где поверхностно односвязная ограниченная область $\Omega \in \mathbb{R}^{3}$ имеет в случае (1.1) гладкую границу $\partial \Omega$ класса $C^{(2, \delta)}$, т.е. $\partial \Omega \in C^{(2, \delta)}$, а в случаях $(1.2)$ и (1.3) $\partial \Omega \in C^{(4, \delta)}$, где $\delta \in(0,1],\left(x_{1}, x_{2}, x_{3}\right) \in \Omega$.

Работа выполнена при финансовой поддержке Российского фонда фундаментальных исследований, гранты № 02-01-00253 и № 02-01-06038, и гранта "Молодые ученые России”. 
Все указанные задачи имеют физический смысл. Вьвод уравнения (1.1) приведен в [1]. Относительно задач (1.2) и (1.3) отметим, что они описывают реальные квазистационарные процессы в полупроводниках и магнетиках соответственно при учете пространственной дисперсии, и мы в ближайшее время опубликуем работу, где будет приведен вьвод соответствующих уравнений.

Уравнение (1.1) является модельным трехмерным обобщением широко известного одномерного модельного уравнения типа Бенджамена-Бона-Махони. Исследованию различных задач для уравнения данного типа посвяшено большое количество работ. Отметим работы [2]-[19]. В этих работах исследуются как одномерные так и многомерные уравнения типа Бенджамена-Бона-Махони и Бенджамена-Бона-Махони-Бюргерса. Изучены вопросы устойчивости решений типа уединенных волн, задачи рассеяния, периодические задачи и асимптотическое поведение при больших временах.

Уравнение (1.2) является модельньм трехмерньм обобщением известного модельного одномерного уравнения типа Розенау. Исследованию как одномерных, так и многомерных уравнений типа Розенау и Розенау-Бюргерса посвящены работы [20]-[26]. В этих работах исследуется асимптотическое поведение решений при больших временах, а также применение метода Галёркина и метода конечно-разностной аппроксимации к исследованию начально-краевых задач для данных уравнений.

Наконец, уравнение (1.3) является, по всей видимости, новым. Заметим, что как волновое уравнение оно является существенно трехмерным. Данное уравнение описьвает квазистационарные волновые процессы в магнетиках: спиновые волны. Работы по математическому исследованию макроскопических (феноменологических) нелинейных моделей теории спиновых волн нам не известны. Отметим, что широкий круг линейных моделей физики спиновых волн был обнаружен нами ранее и опубликован в работе [27].

Для задач (1.1)-(1.3) мы докажем "разрушение" сильных обобщенных решений рассматриваемых задач и получим двусторонние оценки на время "разрушения". Наконец, для задачи (1.1) мы докажем “опрокидывание" “ослабленного" решения задачи и получим оценку сверху на время "опрокидьвания".

2. Локальная разрешимость в сильном обобщенном смысле задач (1.1)(1.3). В данном пункте мы докажем локальную во времени разрешимость в сильном обобщенном смысле каждой из задач (1.1)-(1.3).

Введем сначала некоторые обозначения:

$$
\begin{aligned}
& \mathbf{A}_{1} u \equiv-\Delta u: H_{0}^{1}(\Omega) \rightarrow H^{-1}(\Omega), \quad \mathbf{A}_{2} u \equiv \Delta^{2} u-\Delta u: H_{0}^{2}(\Omega) \rightarrow H^{-2}(\Omega), \\
& \mathbf{F}_{1}(u) \equiv \frac{1}{2} \frac{\partial u^{2}}{\partial x_{1}}: H_{0}^{1}(\Omega) \subset L^{4}(\Omega) \rightarrow H^{-1}(\Omega), \\
& \mathbf{F}_{2}(u) \equiv \beta_{1} \frac{\partial}{\partial x_{1}}\left(\frac{\partial u}{\partial x_{2}} \frac{\partial u}{\partial x_{3}}\right)+\beta_{2} \frac{\partial}{\partial x_{2}}\left(\frac{\partial u}{\partial x_{3}} \frac{\partial u}{\partial x_{1}}\right) \\
&+\beta_{3} \frac{\partial}{\partial x_{3}}\left(\frac{\partial u}{\partial x_{1}} \frac{\partial u}{\partial x_{2}}\right): H_{0}^{2}(\Omega) \rightarrow H^{-2}(\Omega), \\
& \mathbf{F}_{3}(u) \equiv u^{3}: H_{0}^{1}(\Omega) \subset L^{4}(\Omega) \rightarrow L^{4 / 3}(\Omega) \subset H^{-1}(\Omega), \\
& \mathbf{F}_{4}(u) \equiv-\operatorname{div}\left(|\nabla u|^{2} \nabla u\right): H_{0}^{2}(\Omega) \subset W_{0}^{1,4}(\Omega) \rightarrow W^{-1,4 / 3}(\Omega) \subset H^{-2}(\Omega) .
\end{aligned}
$$

С учетом указанных обозначений (2.1)-(2.5) мы можем представить задачи (1.1)-(1.3) в виде задач Коши для абстрактных дифференциальных уравнений первого порядка с 
операторньми коэффициентами в банаховых пространствах:

$$
\begin{array}{ll}
\mathbf{A}_{1} \frac{d u}{d t}=\mathbf{F}_{3}(u)-\mathbf{F}_{1}(u), & u(0)=u_{0} \in H_{0}^{1}(\Omega), \\
\mathbf{A}_{2} \frac{d u}{d t}=\mathbf{F}_{4}(u)-\mathbf{F}_{1}(u), & u(0)=u_{0} \in H_{0}^{2}(\Omega), \\
\mathbf{A}_{2} \frac{d u}{d t}=\mathbf{F}_{4}(u)-\mathbf{F}_{2}(u), & u(0)=u_{0} \in H_{0}^{2}(\Omega) .
\end{array}
$$

Введем следующие нормы и скобки двойственности:

$$
\begin{aligned}
& \|v\|_{+s}-\text { норма в } H_{0}^{s}(\Omega),\|v\|_{-s}^{*}-\text { норма в } H^{-s}(\Omega), s>0 ; \\
& \|v\|_{p}-\text { норма в } L^{p}(\Omega), p \in[1,+\infty] ; \\
& \langle\cdot \cdot \cdot\rangle_{s}-\text { скобки двойственности между } H_{0}^{s}(\Omega) \text { и } H^{-s}(\Omega) ; \\
& (\cdot, \cdot)_{p}-\text { скобки двойственности между } L^{p}(\Omega) \text { и } L^{p^{\prime}}(\Omega), p^{\prime} \equiv p /(p-1) .
\end{aligned}
$$

Перейдем теперь к следующему абстрактному обыкновенному дифференциальному уравнению первого порядка с операторными коэффициентами в банаховом пространстве $V_{0}$ :

$$
\mathbf{A} \frac{d u}{d t}=\mathbf{F}(u), \quad u(0)=u_{0} \in V_{0}
$$

Относительно операторных коэффициентов предположим следующее:

$$
\begin{gathered}
\mathbf{A}: V_{0} \rightarrow V_{0}^{*}, \quad \mathbf{F}: V_{0} \rightarrow V_{0}^{*}, \\
\left\|\mathbf{A} v_{1}-\mathbf{A} v_{2}\right\|_{0}^{*} \geqslant m\left\|v_{1}-v_{2}\right\|_{0}, \quad\left\|\mathbf{F}\left(v_{1}\right)-\mathbf{F}\left(v_{2}\right)\right\|_{0}^{*} \leqslant \mu(R)\left\|v_{1}-v_{2}\right\|_{0},
\end{gathered}
$$

$\|v\|_{0}$ - норма в $V_{0},\|v\|_{0}^{*}-$ норма в $V_{0}^{*}$.

Лемма 1. Для любого $u_{0} \in V_{0}$ найдется такое максимальное $T_{0}>0$, что существует единственное максимальное решение задачи (2.9) класса $и \in$ $C^{(1)}\left([0, T] ; V_{0}\right)$ для любого $T \in\left(0, T_{0}\right)$.

ДокАЗАтЕльство. Рассмотрим эквивалентную $(2.9)$ в классе $C^{(1)}\left([0, T] ; V_{0}\right)$ задачу

$$
\frac{d u}{d t}-\mathbf{G}(u)(t)=0, \quad u(0)=u_{0} \in V_{0}, \quad t \in[0, T], \quad u \in C^{(1)}\left([0, T] ; V_{0}\right),
$$

где оператор $\mathbf{G}(u)=\mathbf{A}^{-1} \mathbf{F}(u)(t), u \in C\left([0, T] ; V_{0}\right)$. Справедлива цепочка соотношений

$$
\begin{aligned}
\lim _{t \rightarrow t_{0}}\left\|\mathbf{G}(u)(t)-\mathbf{G}(u)\left(t_{0}\right)\right\|_{0} & \leqslant m^{-1} \lim _{t \rightarrow t_{0}}\left\|\mathbf{F}(u)(t)-\mathbf{F}(u)\left(t_{0}\right)\right\|_{0}^{*} \\
& \leqslant m^{-1} \mu\left(\left\|u\left(t_{0}\right)\right\|_{0}\right) \lim _{t \rightarrow t_{0}}\left\|u(t)-u\left(t_{0}\right)\right\|_{0} \\
& \leqslant C \lim _{t \rightarrow t_{0}}\left\|u(t)-u\left(t_{0}\right)\right\|_{0}=0 .
\end{aligned}
$$

Значит, $\mathbf{G}: C\left([0, T] ; V_{0}\right) \rightarrow C\left([0, T] ; V_{0}\right)$.

Введем следующее множество:

$$
B_{R}=\left\{u(t) \in C\left([0, T] ; V_{0}\right):\|u\|_{T} \leqslant R\right\},
$$

где

$$
\|v\|_{T} \equiv \sup _{t \in[0, T]}\|v\|_{0}
$$


Докажем, что оператор

$$
\mathbf{U}(u) \equiv u_{0}+\int_{0}^{t} d s \mathbf{G}(u)(s), \quad t \in[0, T],
$$

действует из $B_{R}$ в $B_{R}$ и является сжимающим на $B_{R}$. Действительно, поскольку $\mathbf{G}$ : $C\left([0, T] ; V_{0}\right) \rightarrow C\left([0, T] ; V_{0}\right)$, то очевидно $\mathbf{U}: C\left([0, T] ; V_{0}\right) \rightarrow C^{(1)}\left([0, T] ; V_{0}\right)$. С другой стороны,

$$
\begin{gathered}
\|\mathbf{U}(u)\|_{T} \leqslant\left\|u_{0}\right\|_{0}+\int_{0}^{T} d s\|\mathbf{G}(u)\|_{0}(s) \leqslant\left\|u_{0}\right\|_{0}+T \sup _{t \in[0, T]}\|\mathbf{G}(u)\|_{0}(t), \\
\|\mathbf{G}(u)\|_{0}(t) \leqslant m^{-1}\|\mathbf{F}(u)\|_{0}^{*} \leqslant m^{-1} \mu\left(\|u\|_{0}\right)\|u\|_{0} \leqslant m^{-1} \mu(R)\|u\|_{0} .
\end{gathered}
$$

Поэтому

$$
\|\mathbf{U}\|_{T} \leqslant\left\|u_{0}\right\|+m^{-1} \mu(R) R T .
$$

Пусть $\left\|u_{0}\right\|_{0} \leqslant R / 2, T<m_{0} /(2 \mu(R))$, тогда при достаточно большом $R>0$ и малом $T>0$

$$
\|\mathbf{U}\|_{T} \leqslant R
$$

Таким образом, $\mathbf{U}: B_{R} \rightarrow B_{R}$.

Докажем теперь, что оператор $\mathbf{U}$ сжимающий на $B_{R}$. Пусть $u, v \in B_{R}$, тогда имеем

$$
\begin{aligned}
\|\mathbf{U}(u)-\mathbf{U}(v)\|_{T} & \leqslant \int_{0}^{T} d t\|\mathbf{G}(u)-\mathbf{G}(v)\|_{0} \\
& \leqslant m^{-1} \int_{0}^{T} d t\|\mathbf{F}(u)-\mathbf{F}(v)\|_{0}^{*}(t) \leqslant m^{-1} \mu(R) T\|u-v\|_{T} .
\end{aligned}
$$

Из последнего неравенства при $T<m /(2 \mu(R))$ получаем

$$
\|\mathbf{U}(u)-\mathbf{U}(v)\|_{T}<\frac{1}{2}\|u-v\|_{T} .
$$

Таким образом, оператор $\mathbf{U}$ сжимающий на $B_{R}$. Поэтому в силу теоремы о сжимающем отображении для любого $u_{0} \in V_{0}$ найдется такое $T>0$, что существует единственное решение задачи $(2.11)$ класса $C^{(1)}\left([0, T] ; V_{0}\right)$.

Используя стандартный алгоритм продолжения решения во времени, получим, что существует $T_{0}>0$ и единственное решение задачи $(2.9)$ класса $C^{(1)}\left(\left[0, T_{0}\right) ; V_{0}\right)$. Причем либо $T_{0}<+\infty$ и тогда

$$
\lim _{t \uparrow T_{0}}\|u\|_{0}=+\infty
$$

либо $T_{0}=+\infty$.

Действительно, рассмотрим норму $\psi(T) \equiv\|u(t)\|_{T}$. Функция $\psi(T)$ является монотонно возрастающей как функция переменной $T$. Стало быть, при $T \uparrow T_{0}$ функция $\psi(T)$ имеет либо конечньй, либо бесконечный предел. Предположим, что $\psi(T)$ имеет конечньй предел при $T \uparrow T_{0}$. Рассмотрим вспомогательное интегральное уравнение вида (2.11)

$$
u(x, t)=u\left(x, T^{\prime}\right)+\int_{0}^{t} d s \mathbf{G}(u)(s)
$$


Норма $\|\nabla u\|_{2}^{2}\left(T^{\prime}\right)$ равномерно ограничена по $T^{\prime} \in\left(0, T_{0}\right)$, что позволяет выбрать такое $T^{*} \in\left(0, T_{0}\right)$, что для любого $T^{\prime} \in\left(0, T_{0}\right)$ интегральное уравнение имеет единственное решение класса $u(x, t) \in C^{(1)}\left(\left[0, T^{*}\right] ; V_{0}\right)$. Пусть $T^{\prime}=T_{0}-T^{*} / 2$, обозначим через $v(x, t)$ соответствуюшее решение последнего интегрального уравнения и определим $\widehat{u}(x, t)$ на отрезке $\left[0, T_{0}+T^{*} / 2\right]$ :

$$
\widehat{u}(x, t)=\left\{u(x, t), t \in\left[0, T^{\prime}\right] ; v\left(x, t-T^{\prime}\right), t \in\left[T^{\prime}, T_{0}+T^{*} / 2\right]\right\}
$$

По построению $\widehat{u}(x, t)$ является решением задачи $(1.1)-(1.2)$ на отрезке $\left[0, T_{0}+T^{*} / 2\right]$ и в силу локальной единственности является продолжением функции $u(x, t)$. Это противоречит максимальности отрезка $\left[0, T_{0}\right]$. Полученное противоречие доказывает, что

$$
\lim _{T \uparrow T_{0}}\|u(t)\|_{T}=+\infty
$$

Отсюда сразу же получаем, что

$$
\lim _{t \uparrow T_{0}}\|u(t)\|_{0}=+\infty
$$

Таким образом, для любого $u_{0} \in V$ найдется такое $T_{0}>0$, что существует единственное максимальное решение задачи $(2.9)$ класса $u(t) \in C^{(1)}\left(\left[0, T_{0}\right) ; V_{0}\right)$.

Лемма доказана.

\section{Справедлива следующая}

ТЕОрема 1. Для любых начальных функиий $u_{0}(x)$, принадлежсащих классу $H_{0}^{1}(\Omega)$ в случае задачи (2.6) и классу $H_{0}^{2}(\Omega)$ в случае задач (2.7) и (2.8), найдутся такие максимальные моменты времени $T_{0}$, свои для каждой из задач (2.6)-(2.8), для которых существуют единственные максимальные решения задач (2.6)-(2.8), принадлежашие классу $C^{(1)}\left([0, T] ; H_{0}^{1}(\Omega)\right)$ для задачи $(2.6)$ и классу $C^{(1)}\left([0, T] ; H_{0}^{2}(\Omega)\right)$ для задач (2.7) и (2.8) для любых $T \in\left(0, T_{0}\right)$.

ДокАЗАТЕЛьСТво. Для доказательства данной теоремы нам нужно доказать коэрцитивность операторов $\mathbf{A}_{1}: H_{0}^{1}(\Omega) \rightarrow H^{-1}(\Omega)$ и $\mathbf{A}_{2}: H_{0}^{2}(\Omega) \rightarrow H^{-2}(\Omega)$ и ограниченную Липшиц-непрерывность операторов $\mathbf{F}_{j}(u), j=1, \ldots, 4$, в соответствующих пространствах.

Начнем с доказательства коэрцитивности операторов $\mathbf{A}_{1}$ и $\mathbf{A}_{2}$.

Действительно, справедливы следующие неравенства:

$$
\begin{aligned}
& \left\langle\mathbf{A}_{1} v_{1}-\mathbf{A}_{1} v_{2}, v_{1}-v_{2}\right\rangle_{1}=\left(\nabla v_{1}-\nabla v_{2}, \nabla v_{1}-\nabla v_{2}\right)_{2} \geqslant\left\|v_{1}-v_{2}\right\|_{+1}^{2}, \quad v_{1}, v_{2} \in H_{0}^{1}(\Omega), \\
& \left\langle\mathbf{A}_{2} v_{1}-\mathbf{A}_{2} v_{2}, v_{1}-v_{2}\right\rangle_{2}=\left(\nabla v_{1}-\nabla v_{2}, \nabla v_{1}-\nabla v_{2}\right)_{2}+\left(\Delta v_{1}-\Delta v_{2}, \Delta v_{1}-\Delta v_{2}\right)_{2} \\
& =\left\|v_{1}-v_{2}\right\|_{+1}^{2}+\left\|v_{1}-v_{2}\right\|_{+2}^{2} \geqslant\left\|v_{1}-v_{2}\right\|_{+2}^{2}, \quad v_{1}, v_{2} \in H_{0}^{2}(\Omega) .
\end{aligned}
$$

Из полученных неравенств вытекает существование обратных операторов $\mathbf{A}_{1}^{-1}$ : $H^{-1}(\Omega) \rightarrow H_{0}^{1}(\Omega), \mathbf{A}_{2}^{-1}: H^{-2}(\Omega) \rightarrow H_{0}^{2}(\Omega)$ и Лигшиц-непрерьвность обратных операторов. 
Теперь докажем ограниченную Липшищ-непрерьвность каждого из операторов $\mathbf{F}_{j}(u), j=1, \ldots, 4$. Рассмотрим сначала оператор $\mathbf{F}_{1}(u)$, определенньй формулой $(2.2)$ :

$$
\begin{aligned}
\left\|\mathbf{F}_{1}\left(v_{1}\right)-\mathbf{F}_{1}\left(v_{2}\right)\right\|_{-1}^{*} & \leqslant C\left\|v_{1}^{2}-v_{2}^{2}\right\|_{2} \leqslant C\left(\int_{\Omega} d x\left|v_{1}^{2}-v_{2}^{2}\right|^{2}\right)^{1 / 2} \\
& \leqslant C \sum_{i=1}^{2}\left(\int_{\Omega} d x v_{i}^{4}\right)^{1 / 4}\left\|v_{1}-v_{2}\right\|_{4} \\
& \leqslant R C\left\|v_{1}-v_{2}\right\|_{+1}, \quad v_{i} \in H_{0}^{1}(\Omega), \quad\left\|v_{i}\right\|_{+1} \leqslant R
\end{aligned}
$$

где через $C$ мы обозначаем различные постоянные, не зависящие от $R$ (такого, что $\|v\|_{+1} \leqslant R$. Отсюда сразу же получаем, что

$\left\|\mathbf{F}_{1}\left(v_{1}\right)-\mathbf{F}_{1}\left(v_{2}\right)\right\|_{-2}^{*} \leqslant \mu_{1}(R)\left\|v_{1}-v_{2}\right\|_{+2}, \quad v_{i} \in H_{0}^{2}(\Omega), \quad\left\|v_{i}\right\|_{+2} \leqslant R, \quad \mu_{1}(R)=C R$.

Рассмотрим теперь оператор $\mathbf{F}_{2}(u)$. Справедливо следующее неравенство:

$$
\begin{aligned}
&\left\|\mathbf{F}_{2}\left(v_{1}\right)-\mathbf{F}_{2}\left(v_{2}\right)\right\|_{-2}^{*} \leqslant C\left\|\frac{\partial v_{1}}{\partial x_{2}} \frac{\partial v_{1}}{\partial x_{3}}-\frac{\partial v_{2}}{\partial x_{2}} \frac{\partial v_{2}}{\partial x_{3}}\right\|_{-1}^{*}+C\left\|\frac{\partial v_{1}}{\partial x_{3}} \frac{\partial v_{1}}{\partial x_{1}}-\frac{\partial v_{2}}{\partial x_{3}} \frac{\partial v_{2}}{\partial x_{1}}\right\|_{-1}^{*} \\
&+C\left\|\frac{\partial v_{1}}{\partial x_{2}} \frac{\partial v_{1}}{\partial x_{1}}-\frac{\partial v_{2}}{\partial x_{2}} \frac{\partial v_{2}}{\partial x_{1}}\right\|_{-1}^{*}=J_{1}+J_{2}+J_{3}
\end{aligned}
$$

Рассмотрим, например, слагаемое $J_{1}$, поскольку совершенно понятно, что остальные слагаемые $J_{2}$ и $J_{3}$ оцениваются с точностью до обозначений таким же образом. Действительно,

$$
\begin{aligned}
J_{1} & =C\left\|\frac{\partial v_{1}}{\partial x_{2}} \frac{\partial v_{1}}{\partial x_{3}}-\frac{\partial v_{2}}{\partial x_{2}} \frac{\partial v_{2}}{\partial x_{3}}\right\|_{-1}^{*} \\
& \leqslant C\left\|\frac{\partial v_{1}}{\partial x_{2}}\left[\frac{\partial v_{1}}{\partial x_{3}}-\frac{\partial v_{2}}{\partial x_{3}}\right]\right\|_{-1}^{*}+C\left\|\frac{\partial v_{2}}{\partial x_{3}}\left[\frac{\partial v_{1}}{\partial x_{2}}-\frac{\partial v_{2}}{\partial x_{2}}\right]\right\|_{-1}^{*} \\
& \leqslant C\left(\int_{\Omega} d x\left|\frac{\partial v_{1}}{\partial x_{2}}\right|^{2}\left[\frac{\partial v_{1}}{\partial x_{3}}-\frac{\partial u_{2}}{\partial x_{3}}\right]^{2}\right)^{1 / 2}+C\left(\int_{\Omega} d x\left|\frac{\partial v_{2}}{\partial x_{3}}\right|^{2}\left[\frac{\partial v_{1}}{\partial x_{2}}-\frac{\partial u_{2}}{\partial x_{2}}\right]^{2}\right)^{1 / 2} \\
& \leqslant C \sum_{i=1}^{2}\left\|\nabla v_{i}\right\|_{4}\left\|\nabla v_{1}-\nabla v_{2}\right\|_{4} \leqslant C \sum_{i=1}^{2}\left\|v_{i}\right\|_{+2}\left\|v_{1}-v_{2}\right\|_{+2} \\
& \leqslant C R\left\|v_{1}-v_{2}\right\|_{+2}, \quad v_{i} \in H_{0}^{2}(\Omega), \quad\left\|v_{i}\right\|_{+2} \leqslant R .
\end{aligned}
$$

Таким образом, приходим к выводу, что справедливо следующее неравенство:

$$
\left\|\mathbf{F}_{2}\left(v_{1}\right)-\mathbf{F}_{2}\left(v_{2}\right)\right\|_{-2}^{*} \leqslant \mu_{2}(R)\left\|v_{1}-v_{2}\right\|_{+2}, \quad v_{i} \in H_{0}^{2}(\Omega), \quad \mu_{2}(R)=C R, \quad\left\|v_{i}\right\|_{+2} \leqslant R .
$$


Рассмотрим теперь оператор $\mathbf{F}_{3}(u)$. Справедлива следующая цепочка неравенств:

$$
\begin{aligned}
\left\|\mathbf{F}_{3}\left(v_{1}\right)-\mathbf{F}_{3}\left(v_{2}\right)\right\|_{-1}^{*} & \leqslant C\left\|v_{1}^{3}-v_{2}^{3}\right\|_{4 / 3} \leqslant C\left(\int_{\Omega} d x\left|v_{1}^{3}-v_{2}^{3}\right|^{4 / 3}\right)^{3 / 4} \\
& \leqslant C \sum_{i=1}^{2}\left(\int_{\Omega} d x\left|v_{i}\right|^{8 / 3}\left|v_{1}-v_{2}\right|^{4 / 3}\right)^{3 / 4} \\
& \leqslant C \sum_{i=1}^{2}\left(\int_{\Omega} d x\left|v_{i}\right|^{r_{1} 8 / 3}\right)^{3 /\left(4 r_{1}\right)}\left(\int_{\Omega} d x\left|v_{1}-v_{2}\right|^{r_{2} 4 / 3}\right)^{3 /\left(4 r_{2}\right)} \\
& \leqslant C \sum_{i=1}^{2}\left(\int_{\Omega} d x\left|v_{i}\right|^{4}\right)^{1 / 2}\left(\int_{\Omega} d x\left|v_{1}-v_{2}\right|^{4}\right)^{1 / 4} \\
& \leqslant \mu_{3}(R)\left\|v_{1}-v_{2}\right\|_{+1}, \quad v_{i} \in H_{0}^{1}(\Omega),\left\|v_{i}\right\|_{+1} \leqslant R, \quad \mu_{3}(R)=C R^{2} .
\end{aligned}
$$

Отсюда получаем, что

$\left\|\mathbf{F}_{3}\left(v_{1}\right)-\mathbf{F}_{3}\left(v_{2}\right)\right\|_{-1}^{*} \leqslant \mu_{3}(R)\left\|v_{1}-v_{2}\right\|_{+1}, \quad v_{i} \in H_{0}^{1}(\Omega), \quad\left\|v_{i}\right\|_{+1} \leqslant R, \quad \mu_{3}(R)=C R^{2}$.

Рассмотрим теперь оператор $\mathbf{F}_{4}(u)$. Справедлива следующая цепочка неравенств:

$$
\begin{aligned}
\left\|\mathbf{F}_{4}\left(v_{1}\right)-\mathbf{F}_{4}\left(v_{2}\right)\right\|_{-2}^{*} & \leqslant C\left(\left.\int_{\Omega} d x|| \nabla v_{1}\right|^{2} \nabla v_{1}-\left.\left|\nabla v_{2}\right|^{2} \nabla v_{2}\right|^{4 / 3}\right)^{3 / 4} \\
& \leqslant C \sum_{i=1}^{2}\left(\int_{\Omega} d x\left|\nabla v_{i}\right|^{8 / 3}\left|\nabla v_{1}-\nabla v_{2}\right|^{4 / 3}\right)^{3 / 4} \\
& \leqslant C \sum_{i=1}^{2}\left(\int_{\Omega} d x\left|\nabla v_{i}\right|^{4}\right)^{1 / 2}\left\|v_{1}-v_{2}\right\|_{4}, \\
\left\|\mathbf{F}_{4}\left(v_{1}\right)-\mathbf{F}_{4}\left(v_{2}\right)\right\|_{-2}^{*} & \leqslant \mu_{4}(R)\left\|v_{1}-v_{2}\right\|_{+2}, \quad v_{i} \in H_{0}^{2}(\Omega),\left\|v_{i}\right\|_{+2} \leqslant R, \quad \mu_{4}(R)=C R^{2} .
\end{aligned}
$$

Таким образом, мы доказали ограниченную Липшищ-непрерьвность операторов $\mathbf{F}_{j}(u)$, $j=1, \ldots, 4$, в соответствующих гильбертовых пространствах $H_{0}^{1}(\Omega)$ и $H_{0}^{2}(\Omega)$.

Перейдем теперь к следующему абстрактному обыкновенному дифференциальному уравнению (2.9) первого порядка с операторными коэффициентами в банаховом пространстве $V_{0}$.

Относительно операторных коэффициентов предположим следующее:

$$
\begin{gathered}
\mathbf{A}: V_{0} \rightarrow V_{0}^{*}, \quad \mathbf{F}: V_{0} \rightarrow V_{0}^{*}, \\
\left\|\mathbf{A} v_{1}-\mathbf{A} v_{2}\right\|_{0}^{*} \geqslant m\left\|v_{1}-v_{2}\right\|_{0}, \quad\left\|\mathbf{F}\left(v_{1}\right)-\mathbf{F}\left(v_{2}\right)\right\|_{0}^{*} \leqslant \mu(R)\left\|v_{1}-v_{2}\right\|_{0},
\end{gathered}
$$

$\|v\|_{0}$ - норма в $V_{0},\|v\|_{0}^{*}-$ норма в $V_{0}^{*}$.

Теперь заметим, что из доказанных свойств (2.12)-(2.18) операторных коэффициентов задач (2.6)-(2.8) следует, что эти коэффициенты имеют вид задачи (2.9). Таким образом, для задач (2.6)-(2.8) справедлив результат леммы 1.

Теорема доказана. 
3. "Разрушение" решений задач (1.1)-(1.3). В этом пункте мы докажем, что $T_{0}>0$ из теоремы 1 конечное. Тем самьм, мы докажем, что для каждой из задач (1.1)-(1.3) справедливо предельное равенство

$$
\lim _{t \uparrow T_{0}}\|u\|_{+s}=+\infty,
$$

где $s=1$ для задачи (1.1) и $s=2$ для задач $(1.2),(1.3)$.

Справедлива следующая

ЛЕмма 2. Предположим, что $\Phi(t) \in C^{(2)}\left[0, T_{0}\right)$ при некотором максимальном $T_{0}>0$ в том смысле, что либо $T_{0}=+\infty$, либо $T_{0}<+\infty$. Тогда

$$
\lim _{t \uparrow T_{0}} \Phi(t)=+\infty
$$

причем $\Phi(t)>0 u \Phi^{\prime}(t)>0$ при $t \in\left[0, T_{0}\right)$. Пусть, кроме того, при $t \in\left[0, T_{0}\right)$ справедливо обыкновенное дифференциальное неравенство второго порядка

$$
\Phi^{\prime \prime} \Phi-\alpha\left(\Phi^{\prime}\right)^{2}+\gamma \Phi \Phi^{\prime} \geqslant 0, \quad t \in\left[0, T_{0}\right), \quad \alpha>1, \quad \gamma>0
$$

и справедливо неравенство

$$
\Phi_{0}<\frac{\alpha-1}{\gamma} \Phi_{1}, \quad \Phi_{0} \equiv \Phi(0), \quad \Phi_{1} \equiv \Phi^{\prime}(0) .
$$

Тогда $T_{0} \leqslant T_{2}$, әде

$$
T_{2} \equiv-\frac{1}{\gamma} \ln \left(1-\frac{\gamma}{\alpha-1} \frac{\Phi_{0}}{\Phi_{1}}\right)
$$

и справедливо предельное равенство (3.1).

ДокАЗАтЕльство. Разделим обе части неравенства (3.2) на функцию $\Phi^{1+\alpha}$ и в результате эквивалентных преобразований получим

$$
\left(\frac{\Phi^{\prime}}{\Phi^{\alpha}}\right)^{\prime}+\gamma \frac{\Phi^{\prime}}{\Phi^{\alpha}} \geqslant 0, \quad \alpha>1, \quad \gamma>0 .
$$

Введем обозначение

$$
\Gamma(t) \equiv \frac{\Phi^{\prime}}{\Phi^{\alpha}}
$$

Тогда для введенной функции справедливы неравенства

$$
\begin{gathered}
\Gamma^{\prime}(t)+\Gamma(t) \geqslant 0, \quad \Gamma(t) \geqslant \Gamma_{0} \exp (-\gamma t), \quad \frac{\Phi^{\prime}}{\Phi^{\alpha}} \geqslant \Gamma_{0} \exp (-\gamma t), \\
\frac{1}{1-\alpha}\left(\Phi^{1-\alpha}\right)^{\prime} \geqslant \Gamma_{0} \exp (-\gamma t), \quad \Phi^{1-\alpha} \leqslant \Phi_{0}^{1-\alpha}-\Gamma_{0} \frac{\alpha-1}{\gamma}[1-\exp (-\gamma t)], \\
\Phi \geqslant \frac{1}{\left[\Phi_{0}^{1-\alpha}-\Gamma_{0}((\alpha-1) / \gamma)[1-\exp (-\gamma t)]\right]^{1 /(\alpha-1)}} .
\end{gathered}
$$

Потребуем теперь выполнения неравенства (3.3), тогда (3.5) не может быть вьполнено для всех $t \in \mathbb{R}_{+}^{1}$. Именно, найдется такое $T_{0} \leqslant T_{2}$, где $T_{2}$ определено формулой (3.4), что имеет место предельное равенство (3.1).

Лемма доказана.

Теперь докажем основной результат данного пункта. 
ТЕОРемА 2. Пусть выполнены следующие условия для соответствующих за$\partial a^{\varphi}(1.1),(1.2) u(1.3)$ :

1) $\left\|u_{0}\right\|_{4}^{4}>\left\|\nabla u_{0}\right\|_{2}^{2}+\left\|u_{0}\right\|_{2}^{2}$ для задачи (1.1);

2) $\left\|\nabla u_{0}\right\|_{4}^{4}>C_{1}^{4}(\Omega)\left[\left\|\Delta u_{0}\right\|_{2}^{2}+\left\|\nabla u_{0}\right\|_{2}^{2}\right]$ для задачи (1.2);

3) $\left\|\nabla u_{0}\right\|_{4}^{4}>4 \beta^{2}\left[\left\|\nabla u_{0}\right\|_{2}^{2}+\left\|\Delta u_{0}\right\|_{2}^{2}\right]$ для задачи (1.3),

әде $C_{1}(\Omega)$ - константа наилучшего вложсения такая, что $\|v\|_{4} \leqslant C_{1}(\Omega)\|\nabla v\|_{4}$, $\beta \equiv \sum_{i=1}^{3}\left|\beta_{i}\right|$.

Тогда для любых $u_{0} \in H_{0}^{1}(\Omega)$ в случае задачи (1.1) и для любых $u_{0} \in H_{0}^{2}(\Omega)$ в случае задач (1.2) и (1.3) максимальное $T_{0}$ из теоремы 1 является конечным $и$, тем самым, справедливы следующие предельные равенства для всех задач (1.1)-(1.3):

$$
\lim _{t \uparrow T_{0}}\|u\|_{+s}=+\infty
$$

әде $s=1$ для задачи (1.1) $u s=2$ для задач (1.2) и (1.3). Причем для времен разрушения указанных задач справедливы двусторонние оценки $T_{1} \leqslant T_{0} \leqslant T_{2}$, әде для $T_{1}$ и $T_{2}$ справедливы равенства:

1) для задачи (1.1)

$$
T_{2}=-\ln \left(1-\frac{\left\|\nabla u_{0}\right\|_{2}^{2}+\left\|u_{0}\right\|_{2}^{2}}{\left\|u_{0}\right\|_{4}^{4}}\right), \quad T_{1}=\frac{1}{2 C_{2}^{4}(\Omega)} \frac{1}{\left\|\nabla u_{0}\right\|_{2}^{2}+\left\|u_{0}\right\|_{2}^{2}} ;
$$

2) для задачи (1.2)

$$
T_{2}=-\frac{1}{C_{1}^{4}} \ln \left(1-C_{1}^{4} \frac{\left\|\Delta u_{0}\right\|_{2}^{2}+\left\|\nabla u_{0}\right\|_{2}^{2}}{\left\|\nabla u_{0}\right\|_{4}^{4}}\right), \quad T_{1}=\frac{1}{2 C_{3}^{4}(\Omega)} \frac{1}{\left\|\nabla u_{0}\right\|_{2}^{2}+\left\|\Delta u_{0}\right\|_{2}^{2}} ;
$$

3) для задачи (1.3)

$$
T_{2}=-\frac{1}{4 \beta^{2}} \ln \left(1-4 \beta^{2} \frac{\left\|\Delta u_{0}\right\|_{2}^{2}+\left\|\nabla u_{0}\right\|_{2}^{2}}{\left\|\nabla u_{0}\right\|_{4}^{4}}\right), \quad T_{1}=\frac{1}{2 C_{3}^{4}(\Omega)} \frac{1}{\left\|\nabla u_{0}\right\|_{2}^{2}+\left\|\Delta u_{0}\right\|_{2}^{2}},
$$

әде $C_{2}(\Omega)$ - наилучшая постоянная вложения такая, что $\|v\|_{4} \leqslant C_{2}(\Omega)\|\nabla v\|_{2}$ для любых $v \in H_{0}^{1}(\Omega), C_{3}(\Omega)$ - наилучшая постоянная влохсения такая, что $\|\nabla v\|_{4} \leqslant C_{3}(\Omega)\|\Delta v\|_{2}$ для любы $v \in H_{0}^{2}(\Omega)$.

ДокАЗАТЕЛьСТво. Начнем с доказательства результатов теоремы 2 в случае задачи (1.1).

Из теоремы 1 следует, что для задачи (1.1) при условии $u_{0} \in H_{0}^{1}(\Omega)$ найдется такое максимальное $T_{0}>0$, что существует единственное максимальное решение $u(x, t) \in$ $C^{(1)}\left([0, T] ; H_{0}^{1}(\Omega)\right)$ для любого $T \in\left(0, T_{0}\right)$. Поэтому мы можем умножить уравнение (1.1) сначала на $u(x, t)$, а потом на $u^{\prime}(x, t)$ относительно скобок двойственности между гильбертовыми пространствами $H_{0}^{1}(\Omega)$ и $H^{-1}(\Omega)$. Тогда, интегрируя по частям, получим следуюшие “энергетические” равенства:

$$
\begin{gathered}
\frac{1}{2}\left[\|\nabla u\|_{2}^{2}+\|u\|_{2}^{2}\right]=\|u\|_{4}^{4} \\
\left\|\nabla u^{\prime}\right\|_{2}^{2}+\left\|u^{\prime}\right\|_{2}^{2}=-\frac{1}{2} \int_{\Omega} d x \frac{\partial^{2} u}{\partial x_{1} \partial t} u^{2}+\frac{1}{4} \frac{d}{d t}\|u\|_{4}^{4}
\end{gathered}
$$


Введем обозначение

$$
\Phi_{1}(t) \equiv\|\nabla u\|_{2}^{2}+\|u\|_{2}^{2}
$$

Справедлива следующая цепочка неравенств:

$$
\begin{aligned}
\frac{1}{4}\left|\frac{d \Phi_{1}}{d t}\right|^{2} & \leqslant\left|\int_{\Omega} d x\left[\left(\nabla u^{\prime}, \nabla u\right)+\left(u^{\prime}, u\right)\right]\right|^{2} \\
& \leqslant\left(\left\|\nabla u^{\prime}\right\|_{2}^{2}+\left\|u^{\prime}\right\|_{2}^{2}\right)\left(\|\nabla u\|_{2}^{2}+\|u\|_{2}^{2}\right) \leqslant \Phi_{1}\left(\left\|\nabla u^{\prime}\right\|_{2}^{2}+\left\|u^{\prime}\right\|_{2}^{2}\right) .
\end{aligned}
$$

С другой стороны,

$$
\begin{gathered}
\left\|\nabla u^{\prime}\right\|_{2}^{2}+\left\|u^{\prime}\right\|_{2}^{2} \leqslant \frac{1}{8} \Phi_{1}^{\prime \prime}+\frac{1}{4} \varepsilon\left\|\nabla u^{\prime}\right\|_{2}^{2}+\frac{1}{4} \frac{1}{\varepsilon}\|u\|_{4}^{4} \leqslant \frac{1}{8} \Phi_{1}^{\prime \prime}+\frac{\varepsilon}{4}\left[\left\|\nabla u^{\prime}\right\|_{2}^{2}+\left\|u^{\prime}\right\|_{2}^{2}\right]+\frac{1}{8 \varepsilon} \Phi_{1}^{\prime}, \\
\left(1-\frac{\varepsilon}{4}\right)\left\|\nabla u^{\prime}\right\|_{2}^{2}+\left\|u^{\prime}\right\|_{2}^{2} \leqslant \frac{1}{8} \Phi_{1}^{\prime \prime}+\frac{1}{8 \varepsilon} \Phi_{1}^{\prime}
\end{gathered}
$$

для любого $\varepsilon \in(0,4)$. Из $(3.9)$ и $(3.10)$ вытекают следующие обыкновенные дифференциальные неравенства:

$$
\frac{4-\varepsilon}{16}\left|\Phi_{1}^{\prime}\right|^{2} \leqslant \Phi_{1}\left(\frac{1}{8} \Phi_{1}^{\prime \prime}+\frac{1}{8 \varepsilon} \Phi_{1}^{\prime}\right), \quad \frac{4-\varepsilon}{2}\left|\Phi_{1}^{\prime}\right|^{2} \leqslant \Phi_{1}\left(\Phi_{1}^{\prime \prime}+\frac{1}{\varepsilon} \Phi_{1}^{\prime}\right),
$$

откуда сразу же получаем, что

$$
\Phi_{1} \Phi_{1}^{\prime \prime}-\alpha_{1}\left(\Phi_{1}^{\prime}\right)^{2}+\gamma_{1} \Phi_{1} \Phi_{1}^{\prime} \geqslant 0, \quad \alpha_{1}=\frac{4-\varepsilon}{2}, \quad \gamma_{1}=\frac{1}{\varepsilon}, \quad \varepsilon \in(0,2) .
$$

Мы видим, что функция $\Phi_{1}(t)$ при дополнительном условии

$$
\Phi_{10}<\frac{\alpha_{1}-1}{\gamma_{1}} \Phi_{11}, \quad \Phi_{10} \equiv \Phi_{1}(0), \quad \Phi_{11} \equiv \Phi_{1}^{\prime}(0),
$$

удовлетворяет всем условиям леммы 2. Заметим, что неравенство (3.12) содержит переменную величину $\varepsilon \in(0,2)$. Так что наша задача заключается в получении наиболее оптимального условия вида (3.12). С этой целью нам необходимо найти максимум функции

$$
f_{1}(\varepsilon) \equiv \frac{\alpha_{1}-1}{\gamma_{1}}=\frac{(2-\varepsilon) \varepsilon}{2} .
$$

Максимум этой функции достигается в точке $\varepsilon_{0}=1$ и имеет в этой точке значение $f_{1}\left(\varepsilon_{0}\right)=1 / 2$. Так что условие (3.12) примет вид

$$
\Phi_{10}<\frac{1}{2} \Phi_{11}, \quad \Phi_{10} \equiv \Phi_{1}(0), \quad \Phi_{11} \equiv \Phi_{1}^{\prime}(0),
$$

откуда с учетом определения $\Phi_{1}(t)$ приходим к условию 1$)$ теоремы 2 . Теперь рассмотрим дифференциальное неравенство (3.11) при $\varepsilon=\varepsilon_{0}=1$. Из (3.11) получим

$$
\Phi_{1} \Phi_{1}^{\prime \prime}-\alpha_{1}\left(\Phi_{1}^{\prime}\right)^{2}+\gamma_{1} \Phi_{1} \Phi_{1}^{\prime} \geqslant 0, \quad \alpha_{1}=\frac{3}{2}, \quad \gamma_{1}=1 .
$$

Откуда в силу результата леммы 2 получим, что $T_{0} \leqslant T_{2}$, где

$$
T_{2}=-\ln \left(1-\frac{\left\|\nabla u_{0}\right\|_{2}^{2}+\left\|u_{0}\right\|_{2}^{2}}{\left\|u_{0}\right\|_{4}^{4}}\right) .
$$


Переходим к рассмотрению задачи (1.2).

Из теоремы 1 следует, что для задачи $(1.2)$ при условии $u_{0} \in H_{0}^{2}(\Omega)$ найдется такое максимальное $T_{0}>0$, что существует единственное максимальное решение $u(x, t) \in$ $C^{(1)}\left([0, T] ; H_{0}^{2}(\Omega)\right)$ для любого $T \in\left(0, T_{0}\right)$. Поэтому мы можем умножить уравнение $(1.2)$ сначала на $u(x, t)$, а потом на $u^{\prime}(x, t)$ относительно скобок двойственности между гильбертовыми пространствами $H_{0}^{2}(\Omega)$ и $H^{-2}(\Omega)$. Тогда, интегрируя по частям, получим следующие "энергетические" равенства:

$$
\begin{gathered}
\frac{1}{2} \frac{d}{d t}\left[\|\nabla u\|_{2}^{2}+\|\Delta u\|_{2}^{2}\right]=\|\nabla u\|_{4}^{4}, \\
\left\|\nabla u^{\prime}\right\|_{2}^{2}+\|\Delta u\|_{2}^{2}=\frac{1}{4} \frac{d}{d t}\|\nabla u\|_{4}^{4}-\frac{1}{2} \int_{\Omega} d x \frac{\partial^{2} u}{\partial x_{1} \partial t} u^{2} .
\end{gathered}
$$

Из равенства (3.16) с учетом (3.15) вытекает следующее неравенство:

$$
\begin{aligned}
\left\|\nabla u^{\prime}\right\|_{2}^{2}+\|\Delta u\|_{2}^{2} & \leqslant \frac{1}{4} \frac{d}{d t}\|\nabla u\|_{4}^{4}+\frac{1}{4} \varepsilon\left[\left\|\nabla u^{\prime}\right\|_{2}^{2}+\|\Delta u\|_{2}^{2}\right]+\frac{1}{4 \varepsilon}\|u\|_{4}^{4} \\
& \leqslant \frac{1}{4} \frac{d}{d t}\|\nabla u\|_{4}^{4}+\frac{1}{4} \varepsilon\left[\left\|\nabla u^{\prime}\right\|_{2}^{2}+\|\Delta u\|_{2}^{2}\right]+\frac{1}{4 \varepsilon} C_{1}^{4}(\Omega)\|\nabla u\|_{4}^{4} \quad \forall \varepsilon \in(0,4),
\end{aligned}
$$

где $C_{1}$ - наилучшая постоянная вложения такая, что $\|v\|_{4} \leqslant C_{1}(\Omega)\|\nabla v\|_{4}$. Введем обозначение

$$
\Phi_{2}(t) \equiv\|\nabla u\|_{2}^{2}+\|\Delta u\|_{2}^{2} .
$$

Из (3.15)-(3.17) вытекает следующее обыкновенное дифференциальное неравенство для функции $\Phi_{2}(t)$ :

$$
\frac{4-\varepsilon}{16}\left(\Phi_{2}^{\prime}\right)^{2} \leqslant \Phi_{2}\left[\frac{1}{8} \Phi_{2}^{\prime \prime}+\frac{C_{1}^{4}(\Omega)}{8 \varepsilon} \Phi_{2}^{\prime}\right],
$$

из которого вытекает такое дифференциальное неравенство:

$$
\Phi_{2} \Phi_{2}^{\prime \prime}-\alpha_{2}\left(\Phi_{2}^{\prime}\right)^{2}+\gamma_{2} \Phi_{2} \Phi_{2}^{\prime} \geqslant 0, \quad \alpha_{2} \equiv \frac{4-\varepsilon}{2}, \quad \gamma_{2} \equiv \frac{C_{1}^{4}(\Omega)}{\varepsilon} .
$$

Поступая теперь точно так же, как и в случае дифференциального неравенства (3.11), мы приходим к условию леммы 2 :

$$
\Phi_{20}<\frac{\alpha_{2}-1}{\gamma_{2}} \Phi_{21}, \quad \Phi_{20} \equiv \Phi_{2}(0), \quad \Phi_{21} \equiv \Phi_{2}^{\prime}(0) .
$$

Выбираем $\varepsilon$ из условия экстремума функции

$$
f_{2}(\varepsilon)=\frac{\alpha_{2}-1}{\gamma_{2}}=\frac{(2-\varepsilon) \varepsilon}{2 C_{1}^{4}},
$$

минимум которой достигается в точке $\varepsilon_{0}=1$ и имеет значение

$$
f_{2}\left(\varepsilon_{0}\right)=\frac{1}{2 C_{1}^{4}(\Omega)}
$$


Из (3.18) при $\varepsilon=1$ приходим к следующему дифференциальному неравенству:

$$
\Phi_{2} \Phi_{2}^{\prime \prime}-\alpha_{2}\left(\Phi_{2}^{\prime}\right)^{2}+\gamma_{2} \Phi_{2} \Phi_{2}^{\prime} \geqslant 0, \quad \alpha_{2} \equiv \frac{3}{2}, \quad \gamma_{2} \equiv C_{1}^{4}(\Omega)
$$

а условие (3.19) при $\varepsilon=1$ принимает вид

$$
\left\|\nabla u_{0}\right\|_{4}^{4}>C_{1}^{4}(\Omega)\left[\left\|\Delta u_{0}\right\|_{2}^{2}+\left\|\nabla u_{0}\right\|_{2}^{2}\right] .
$$

Из леммы 2 вытекает условие $T_{0} \leqslant T_{2}$, где

$$
T_{2}=-\frac{1}{C_{1}^{4}} \ln \left(1-C_{1}^{4} \frac{\left\|\Delta u_{0}\right\|_{2}^{2}+\left\|\nabla u_{0}\right\|_{2}^{2}}{\left\|\nabla u_{0}\right\|_{4}^{4}}\right) .
$$

Рассмотрим теперь задачу (1.3).

Из теоремы 1 следует, что для задачи (1.3) при условии $u_{0} \in H_{0}^{2}(\Omega)$ найдется такое максимальное $T_{0}>0$ и существует единственное максимальное решение $u(x, t) \in$ $C^{(1)}\left([0, T] ; H_{0}^{2}(\Omega)\right)$ для любого $T \in\left(0, T_{0}\right)$. Поэтому мы можем умножить уравнение (1.3) сначала на $u(x, t)$, а потом на $u^{\prime}(x, t)$ относительно скобок двойственности между гильбертовыми пространствами $H_{0}^{2}(\Omega)$ и $H^{-2}(\Omega)$. Тогда, интегрируя по частям, получим следующие “энергетические” равенства:

$$
\begin{aligned}
& \frac{1}{2} \frac{d}{d t}\left[\|\nabla u\|_{2}^{2}+\|\Delta u\|_{2}^{2}\right]=\|\nabla u\|_{4}^{4} \\
\left\|\Delta u^{\prime}\right\|_{2}^{2}+\|\nabla u\|_{2}^{2}= & \frac{1}{4} \frac{d}{d t}\|\nabla u\|_{4}^{4}-\beta_{1} \int_{\Omega} d x \frac{\partial^{2} u}{\partial x_{1} \partial t} \frac{\partial u}{\partial x_{2}} \frac{\partial u}{\partial x_{3}} \\
& -\beta_{2} \int_{\Omega} d x \frac{\partial^{2} u}{\partial x_{2} \partial t} \frac{\partial u}{\partial x_{3}} \frac{\partial u}{\partial x_{1}}-\beta_{3} \int_{\Omega} d x \frac{\partial^{2} u}{\partial x_{3} \partial t} \frac{\partial u}{\partial x_{1}} \frac{\partial u}{\partial x_{2}}
\end{aligned}
$$

Из (3.21) и (3.22) вытекает следующая цепочка неравенств:

$$
\begin{aligned}
\left\|\Delta u^{\prime}\right\|_{2}^{2}+\left\|\nabla u^{\prime}\right\|_{2}^{2} & \leqslant \frac{1}{4} \frac{d}{d t}\|\nabla u\|_{4}^{4}+\sum_{i=1}^{3}\left|\beta_{i}\right|\left\|\nabla u^{\prime}\right\|_{2}\|\nabla u\|_{4}^{2} \\
& \leqslant \frac{1}{4} \frac{d}{d t}\|\nabla u\|_{4}^{4}+\sum_{i=1}^{3}\left|\beta_{i}\right| \frac{\varepsilon}{2}\left[\left\|\nabla u^{\prime}\right\|_{2}^{2}+\left\|\Delta u^{\prime}\right\|_{2}^{2}\right]+\frac{1}{2 \varepsilon} \sum_{i=1}^{3}\left|\beta_{i}\right|\|\nabla u\|_{4}^{4}
\end{aligned}
$$

где

$$
\varepsilon \in\left(0,2 \beta^{-1}\right), \quad \beta=\sum_{i=1}^{3}\left|\beta_{i}\right|>0
$$

Введем обозначение

$$
\Phi_{3}(t) \equiv\|\nabla u\|_{2}^{2}+\|\Delta u\|_{2}^{2}
$$

Из (3.21)-(3.23) получим обыкновенное дифференциальное неравенство для функции $\Phi_{3}(t)$ :

$$
\Phi_{3} \Phi_{3}^{\prime \prime}-\alpha_{3}\left(\Phi_{3}^{\prime}\right)^{2}+\gamma_{3} \Phi_{3} \Phi_{3}^{\prime} \geqslant 0, \quad \alpha_{3}=2-\varepsilon \beta, \quad \gamma_{3}=\frac{2 \beta}{\varepsilon} .
$$


Точно так же, как и раньше, используя результаты леммы 2 , из (3.24) получим оптимальное условие на начальную функцию

$$
\left\|\nabla u_{0}\right\|_{4}^{4}>4 \beta^{2}\left[\left\|\nabla u_{0}\right\|_{2}^{2}+\left\|\Delta u_{0}\right\|_{2}^{2}\right]
$$

и оценку сверху на время разрушения $T_{0} \leqslant T_{2}$ :

$$
T_{2}=-\frac{1}{4 \beta^{2}} \ln \left(1-4 \beta^{2} \frac{\left\|\Delta u_{0}\right\|_{2}^{2}+\left\|\nabla u_{0}\right\|_{2}^{2}}{\left\|\nabla u_{0}\right\|_{4}^{4}}\right) .
$$

Получим теперь оценки снизу на время разрушения решений задач (1.1)-(1.3).

Для задачи (1.1) справедливо энергетическое равенство

$$
\frac{1}{2} \frac{d}{d t}\left(\|\nabla u\|_{2}^{2}+\|u\|_{2}^{2}\right)=\|u\|_{4}^{4} .
$$

Откуда для функции

$$
\Phi_{1}(t) \equiv\|\nabla u\|_{2}^{2}+\|u\|_{2}^{2}
$$

вытекает неравенство

$$
\Phi_{1}(t) \leqslant \Phi_{1}(0)+2 C_{2}^{4}(\Omega) \int_{0}^{t} d s \Phi_{1}^{2}(s),
$$

где $C_{2}$ - наилучшая постоянная вложения такая, что $\|v\|_{4} \leqslant C_{2}(\Omega)\|\nabla v\|_{2}$ для любых $v \in H_{0}^{1}(\Omega)$, из которого вытекает

$$
\Phi_{1}(t) \leqslant \frac{\Phi_{1}(0)}{1-2 C_{2}^{4}(\Omega) \Phi_{1}(0) t}
$$

и, стало быть, в данном случае

$$
T_{1}=\frac{1}{2 C_{2}^{4}(\Omega)} \frac{1}{\left\|\nabla u_{0}\right\|_{2}^{2}+\left\|u_{0}\right\|_{2}^{2}} .
$$

Для задач (1.2) и (1.3) справедливо энергетическое равенство

$$
\frac{1}{2} \frac{d}{d t}\left(\|\Delta u\|_{2}^{2}+\|\nabla u\|_{2}^{2}\right)=\|\nabla u\|_{4}^{4},
$$

и тогда для функции

$$
\Phi_{2}(t) \equiv\|\Delta u\|_{2}^{2}+\|\nabla u\|_{2}^{2}
$$

справедливо неравенство

$$
\Phi_{2}(t) \leqslant \Phi_{2}(0)+2 C_{3}^{4}(\Omega) \int_{0}^{t} d s \Phi_{2}^{2}(s),
$$

где $C_{3}(\Omega)$ - наилучшая постоянная вложения такая, что $\|\nabla v\|_{4} \leqslant C_{3}(\Omega)\|\Delta v\|_{2}$ для любых $v \in H_{0}^{2}(\Omega)$. Отсюда сразу же получаем, что

$$
T_{1}=\frac{1}{2 C_{3}^{4}(\Omega)} \frac{1}{\left\|\nabla u_{0}\right\|_{2}^{2}+\left\|\Delta u_{0}\right\|_{2}^{2}} .
$$

Теорема доказана. 
4. “Опрокидывание" “ослабленного” решения задачи (1.1). Для доказательства “опрокидывания" решения задачи (1.1) для трехмерного уравнения типа Бенджамена-Бона-Махони нам необходимо доказать, что существует такое максимальное $T_{0}^{*}$, что существует единственное максимальное решение задачи $(1.1)$ класса $C^{(1)}([0, T]$; $\left.C^{(1)}(\bar{\Omega})\right)$ для любого $T \in\left(0, T_{0}^{*}\right)$. Тогда в силу результата п. 3 о "разрушении" мы получим, что $0<T_{0}^{*} \leqslant T_{0} \leqslant T_{2}<+\infty$, а значит,

$$
\lim _{T \uparrow T_{0}^{*}} \sup _{t \in[0, T], x \in \bar{\Omega}}|\nabla u(x, t)|=+\infty
$$

т.е. в некоторьй момент времени $T_{0}^{*}>0$ величина

$$
\max _{x \in \bar{\Omega}}|\nabla u(x, t)|
$$

становится равной бесконечности. В этом пункте под решением задачи (1.1) мы будем понимать так назьваемое "ослабленное" решение, т.е. решение некоторого связанного с задачей (1.1) интегрального уравнения, вид которого будет установлен ниже. Причем классическое решение задачи (1.1) является “ослабленным", обратное утверждение, очевидно, имеет место не всегда.

Дадим определение “ослабленного” решения задачи (1.1).

ОПРеДЕЛЕниЕ 1. “Ослабленным" решением задачи $(1.1)$ мы назовем решение $u(x, t)$ класса $C^{(1)}\left([0, T] ; C^{(1)}(\bar{\Omega}) \cap C_{0}(\bar{\Omega})\right)$ при некотором $T>0$ следующего двойного интегрального уравнения:

$$
u(x, t)=u_{0}(x)+\int_{0}^{t} d s \int_{\Omega} d y G(x, y)\left[\frac{1}{2} \frac{\partial u^{2}(y, s)}{\partial y_{1}}+u^{3}(y, s)\right], \quad t \in[0, T],
$$

где $G(x, y)$ - функция Грина первой краевой задачи для оператора $-\Delta+\mathbf{I}$ в области $\Omega$.

Очевидно, с необходимостью получаем, что для существования "ослабленного" решения необходимо условие на начальную функцию $u_{0}(x) \in C^{(1)}(\bar{\Omega}) \cap C_{0}(\bar{\Omega})$.

Теперь мы выясним как связаны сильные обобщенные решения задачи (1.1) и "ослабленные” решения в смысле сформулированного определения. Как мы обнаружили в п. 3, решение задачи (1.1), понимаемое в сильном обобщенном смысле, эквивалентно решению интегрального операторного уравнения вида

$$
u(t)=u_{0}+\int_{0}^{t} d s \mathbf{A}^{-1} \mathbf{F}(u), \quad u_{0} \in H_{0}^{1},
$$

где оператор $\mathbf{A}^{-1}: H^{-1}(\Omega) \rightarrow H_{0}^{1}(\Omega)$ является обратным к оператору $-\Delta u+u: H_{0}^{1}(\Omega)$ $\rightarrow H^{-1}(\Omega)$. Тогда понятно, что сужение оператора $\mathbf{A}^{-1}$ на банахово пространство $C(\bar{\Omega})$, где $\Omega$ имеет гладкую границу $\partial \Omega \in C^{(2, \delta)}, \delta \in(0,1]$, совпадает с интегральным оператором, ядро которого является функцией Грина $G(x, y)$. Таким образом, мы приходим к выводу, что "ослабленное" решение задачи (1.1) является сильным обобщенным решением задачи (1.1). Этот результат нам понадобится для доказательства “опрокидывания" “ослабленного" решения задачи (1.1).

ТЕорема 3. Для любого $u_{0} \in C^{(1)}(\Omega) \cap C_{0}(\bar{\Omega})$ найдется такое максимальное $T_{0}^{*}>0$, что существует единственное максимальное "ослабленное" решение $u(x, t)$ класса $C^{(1)}\left([0, T] ; C^{(1)}(\bar{\Omega}) \cap C_{0}(\bar{\Omega})\right)$ для любого $T \in\left(0, T_{0}^{*}\right)$. Причем либо $T_{0}^{*}=+\infty$, либо $T_{0}^{*}<+\infty$, и тогда справедливо предельное соотношение (4.1). 
ДокАЗАТЕЛЬСтво. В силу того, что функция Грина для оператора $-\Delta+\mathbf{I}$ имеет вид

$$
G(x, y)=\psi(x, y)+\frac{1}{4 \pi} \frac{\exp (-|x-y|)}{|x-y|}
$$

где $\psi(x, y) \in C^{(1)}(\bar{\Omega} \times \bar{\Omega})$, справедливо соотношение

$$
\sup _{x \in \Omega} \int_{\Omega}\left|\nabla_{y} G(x, y)\right| d y=C<+\infty .
$$

Поэтому мы можем проинтегрировать по частям интегродифференциальное уравнение (4.2) и получить следующее соотношение:

$$
u(x, t)=u_{0}(x)+\int_{0}^{t} d s \int_{\Omega} d y G(x, y) u^{3}(y, s)-\frac{1}{2} \int_{0}^{t} d s \int_{\Omega} d y G_{y_{1}}^{\prime}(x, y) u^{2} .
$$

Введем оператор

$$
\mathbf{U}(u) \equiv u_{0}+\int_{0}^{t} d s \int_{\Omega} d y G(x, y) u^{3}(y, s)-\frac{1}{2} \int_{0}^{t} d s \int_{\Omega} d y G_{y_{1}}^{\prime}(x, y) u^{2} .
$$

Докажем, что оператор (4.6) действует из $C\left(\bar{Q}_{T}\right)$ в $C\left(\bar{Q}_{T}\right)$, где $Q_{T}=(0, T) \times \Omega$ при некотором $T>0$ и

$$
\|v\|_{T, 0}=\sup _{t \in[0, T], x \in \Omega}|v|
$$

- норма в $C\left(\bar{Q}_{T}\right)$.

Введем обозначения

$$
\mathbf{U}_{1}(u)=\int_{0}^{t} d s \int_{\Omega} d y G_{y_{1}}^{\prime}(x, y) u^{2}, \quad \mathbf{U}_{2}(u)=\int_{0}^{t} d s \int_{\Omega} d y G(x, y) u^{3}(y, s) .
$$

Докажем, что каждый из операторов $\mathbf{U}_{i}(u): C\left(\bar{Q}_{T}\right) \rightarrow C\left(\bar{Q}_{T}\right)$.

Рассмотрим сначала оператор $\mathbf{U}_{1}(u)$. Действительно,

$$
\begin{gathered}
\psi_{y_{1}}^{\prime}(x, y) \in C(\Omega \times \Omega), \\
\frac{1}{4 \pi} \frac{\partial}{\partial y_{1}}\left(\frac{\exp (-|x-y|)}{|x-y|}\right)=\frac{1}{4 \pi} \frac{x_{1}-y_{1}}{|x-y|^{3}} \exp (-|x-y|)+\frac{1}{4 \pi} \frac{x_{1}-y_{1}}{|x-y|^{2}} \exp (-|x-y|),
\end{gathered}
$$

тогда $\mathbf{U}_{1}(u)$ можно представить в виде

$$
\mathbf{U}_{1}(u)=I_{1}+I_{2}+I_{3},
$$

где

$$
\begin{aligned}
& I_{1}=\int_{0}^{t} d s \int_{\Omega} d y \psi_{y_{1}}^{\prime}(x, y) u^{2}(y, s), \\
& I_{2}=\frac{1}{4 \pi} \int_{0}^{t} d s \int_{\Omega} d y \frac{\exp (-|x-y|)}{|x-y|^{2}} \frac{x_{1}-y_{1}}{|x-y|} u^{2}(y, s), \\
& I_{3}=\frac{1}{4 \pi} \int_{0}^{t} d s \int_{\Omega} d y \frac{\exp (-|x-y|)}{|x-y|} \frac{x_{1}-y_{1}}{|x-y|} u^{2}(y, s) .
\end{aligned}
$$


Докажем, что $I_{1}(x, t) \in C\left(\bar{Q}_{T}\right)$. Действительно, для любых $(x, t)$ и $\left(x_{0}, t_{0}\right)$ из $Q_{T}$ справедливо неравенство

$$
\begin{aligned}
\left|I_{1}(x, t)-I_{2}\left(x_{0}, t_{0}\right)\right| \leqslant & \left|\int_{t_{0}}^{t} d s \int_{\Omega} d y\right| \psi_{y_{1}}^{\prime}(x, y)\left|u^{2}\right| \\
& +\int_{0}^{t_{0}} d s\left|\int_{\Omega} d y\left[\psi_{y_{1}}^{\prime}(x, y)-\psi_{y_{1}}^{\prime}\left(x_{0}, y\right)\right] u^{2}\right|
\end{aligned}
$$

откуда в силу непрерьвности $\psi_{y_{1}}^{\prime}(x, y) \in C(\bar{\Omega} \times \bar{\Omega})$ сразу же получаем, что каждое из слагаемых может быть сделано меньше $\varepsilon / 2$ при условии $\left|x-x_{0}\right|+\left|t-t_{0}\right| \leqslant \delta(\varepsilon)$. Значит, $I_{1}(x, t) \in C\left(\bar{Q}_{T}\right)$.

Докажем теперь, что $I_{2}(x, t) \in C\left(\bar{Q}_{T}\right)$. Действительно, для любых $(x, t)$ и $\left(x_{0}, t_{0}\right)$ из $\bar{Q}_{T}$ справедливо неравенство

$$
\begin{aligned}
& \left|I_{2}(x, t)-I_{2}\left(x_{0}, t_{0}\right)\right| \\
& \qquad \leqslant \frac{1}{4 \pi}\left|\int_{t_{0}}^{t} d s \int_{\Omega} d y \frac{\exp (-|x-y|)}{|x-y|^{2}} u^{2}(y, s)\right| \\
& \quad+\frac{1}{4 \pi} \int_{0}^{t_{0}} d s\left|\int_{\Omega} d y\left[\frac{\exp \left(-\left|x_{0}-y\right|\right)}{\left|x_{0}-y\right|^{2}} \frac{x_{10}-y_{1}}{\left|x_{0}-y\right|}-\frac{\exp (-|x-y|)}{|x-y|^{2}} \frac{x_{1}-y_{1}}{|x-y|}\right] u^{2}(y, s)\right| \\
& \quad=I_{21}+I_{22} .
\end{aligned}
$$

Рассмотрим отдельно каждое из слагаемых $I_{21}$ и $I_{22}$. Выражение $I_{21}$ может быть сделано меньше $\varepsilon / 3$ при условии $\left|x-x_{0}\right|+\left|t-t_{0}\right| \leqslant \delta(\varepsilon)$. Рассмотрим теперь $I_{22}$. С этой целью предположим, что $u(x, t) \in C\left(\bar{Q}_{T}\right)$ и $|u(x, t)| \leqslant M(T)$. Далее воспользуемся схемой доказательства непрерывности интеграла типа потенциала, изложенной, например, в [28]. Действительно,

$$
\begin{aligned}
& \left|I_{22}\left(x, x_{0}, t, t_{0}\right)\right| \leqslant M(T) \int_{0}^{t_{0}} d s \int_{\Omega_{\eta}\left(x_{0}\right)} d y\left[\frac{\exp \left(-\left|x_{0}-y\right|\right)}{\left|x_{0}-y\right|^{2}}+\frac{\exp (-|x-y|)}{|x-y|^{2}}\right] \\
& \left.\quad+M(T) \int_{0}^{t_{0}} d s \int_{\Omega_{\eta}\left(x_{0}\right)} \mid \frac{\exp \left(-\left|x_{0}-y\right|\right)}{\left|x_{0}-y\right|^{2}} \frac{x_{10}-y_{1}}{\left|x_{0}-y\right|}-\frac{\exp (-|x-y|)}{|x-y|^{2}} \frac{x_{1}-y_{1}}{|x-y|}\right] .
\end{aligned}
$$

Первое слагаемое в силу интегрируемой особенности подьнтегральной функции может быть сделано меньше $\varepsilon / 3$ за счет выбора малого параметра $\eta$. Во втором слагаемом подынтегральная функция равномерно непрерьвна по $(x, y)$ в области $\left|x-x_{0}\right| \leqslant \eta / 2$, $\left|y-x_{0}\right| \geqslant \eta, y \in \bar{\Omega}$ и обращается в нуль при $x=x_{0}$. Поэтому этот интеграл может быть сделан меньше $\varepsilon / 3$ при всех $\left|x-x_{0}\right|+\left|t-t_{0}\right| \leqslant \delta(\varepsilon)$ при достаточно малом $\delta(\varepsilon)$. Значит, $I_{2}(x, t) \in C\left(\bar{Q}_{T}\right)$.

Совершенно понятно, что точно так же доказывается включение $I_{3}(x, t) \in C\left(\bar{Q}_{T}\right)$. Стало быть, мы доказали, что

$$
\mathbf{U}_{1}(u): C\left(\bar{Q}_{T}\right) \rightarrow C\left(\bar{Q}_{T}\right) .
$$

Рассмотрим теперь оператор

$$
\mathbf{U}_{2}(u)=\int_{0}^{t} d s \int_{\Omega} d y G(x, y) u^{3}(y, s) .
$$


Используя, как и ранее, свойства функции Грина и интегралов типа потенциала, получим, что

$$
\mathbf{U}_{2}: C\left(\bar{Q}_{T}\right) \rightarrow C\left(\bar{Q}_{T}\right)
$$

Стало быть, оператор $\mathbf{U}(u)(x, t)$, определенньй формулой $(4.6)$, действует из $C\left(\bar{Q}_{T}\right)$ в $C\left(\bar{Q}_{T}\right)$.

Пусть $B_{R}$ - замкнутое ограниченное вьпуклое подмножество банахова пространства $C\left(\bar{Q}_{T}\right)$ :

$$
B_{R} \equiv\left\{v(x, t) \in C\left(\bar{Q}_{T}\right):\|v\|_{T} \equiv \max _{(x, t) \in \bar{Q}_{T}}|v(x, t)| \leqslant R\right\} .
$$

Докажем, что при достаточно малом $T>0$ и достаточно большом $R>0$ оператор $\mathbf{U}$ действует из $B_{R}$ в $B_{R}$ и является сжимающим на $B_{R}$. Действительно,

$$
\|\mathbf{U}(u)\|_{T} \leqslant\left\|u_{0}\right\|_{T}+C T\|u\|_{T}^{3}+C T\|u\|_{T}^{2} \leqslant\left\|u_{0}\right\|_{T}+C T\left(R^{3}+R^{2}\right) \leqslant R,
$$

при условии $\left\|u_{0}\right\|_{T} \leqslant R / 2$ и $0<T \leqslant 2^{-1}\left(C R^{3}+C R^{2}\right)^{-1}$. Тем самым, при достаточно малом $T>0$ и достаточно большом $R>0$ оператор $\mathbf{U}$ действует из $B_{R}$ в $B_{R}$.

Докажем теперь сжимаемость оператора на $B_{R}$ при достаточно малом $T>0$ и достаточно большом $R>0$. Действительно,

$$
\left\|\mathbf{U}\left(u_{1}\right)-\mathbf{U}\left(u_{2}\right)\right\|_{T} \leqslant T\left(C R^{2}+C R\right)\left\|u_{1}-u_{2}\right\|_{T} \leqslant \frac{1}{2}\left\|u_{1}-u_{2}\right\|_{T},
$$

при условии $0<T \leqslant 2^{-1}\left(C R^{2}+C R\right)^{-1}$. Значит, оператор $\mathbf{U}(u)$ сжимающий на $B_{R}$.

Стало быть, при условии $u_{0} \in C_{0}(\bar{\Omega})$ сушествует единственное решение интегрального уравнения $(4.5)$ класса $C\left([0, T] ; C_{0}(\bar{\Omega})\right)$.

Теперь предположим, что $u_{0} \in C^{(1)}(\bar{\Omega}) \cap C_{0}(\bar{\Omega})$. Докажем, что существует единственное решение интегрального уравнения $(4.5)$ класса $C\left([0, T] ; C^{(1)}(\bar{\Omega}) \cap C_{0}(\bar{\Omega})\right)$.

С этой целью рассмотрим банахово пространство $C\left([0, T] ; C^{(1)}(\bar{\Omega})\right)$ с нормой

$$
\|v\|_{T, 1} \equiv\|v\|_{T, 0}+\sum_{i=1}^{3}\left\|\frac{\partial v}{\partial x_{i}}\right\|_{T, 0}, \quad\|v\|_{T, 0} \equiv \max _{(x, t) \in \bar{Q}_{T}}|v(x, t)| .
$$

Докажем, что оператор

$$
\mathbf{U}(u) \equiv u_{0}+\int_{0}^{t} d s \int_{\Omega} d y G(x, y)\left[\frac{1}{2} \frac{\partial u^{2}(y, s)}{\partial y_{1}}+u^{3}(y, s)\right],
$$

действует из $C\left([0, T] ; C^{(1)}(\bar{\Omega})\right)$ в $C\left([0, T] ; C^{(1)}(\bar{\Omega})\right)$. С этой целью воспользуемся тем, что

$$
G(x, y)=\psi(x, y)+\frac{1}{4 \pi} \frac{\exp (-|x-y|)}{|x-y|}
$$

и $\psi_{x_{1}}^{\prime}(x, y) \in C(\bar{\Omega} \times \bar{\Omega})$, и представим оператор $\mathbf{U}(u)$ в виде

$$
\mathbf{U}(u)=u_{0}+\mathbf{U}_{1}(u)+\mathbf{U}_{2}(u)
$$

где

$$
\begin{aligned}
& \mathbf{U}_{1}(u)=\int_{0}^{t} \int_{\Omega} d y \psi(x, y)\left[u \frac{\partial u}{\partial y_{1}}+u^{3}\right] \\
& \mathbf{U}_{2}(u)=\frac{1}{4 \pi} \int_{0}^{t} \int_{\Omega} d y \frac{\exp (-|x-y|)}{|x-y|}\left[u \frac{\partial u}{\partial y_{1}}+u^{3}\right] .
\end{aligned}
$$


В силу того, что $\psi(x, y) \in C^{(1)}(\bar{\Omega} \times \bar{\Omega})$, оператор $\mathbf{U}_{1}(u)$ действует из $C\left([0, T] ; C^{(1)}(\bar{\Omega})\right)$ в $C\left([0, T] ; C^{(1)}(\bar{\Omega})\right)$.

Рассмотрим теперь отдельно оператор $\mathbf{U}_{2}(u)$. Точно так же, как и ранее, используя теоремы о непрерывной дифференцируемости интегралов типа потенциала, приходим к выводу, что и оператор $\mathbf{U}_{2}(u)$ действует из $C\left([0, T] ; C^{(1)}(\bar{\Omega})\right)$ в $C\left([0, T] ; C^{(1)}(\bar{\Omega})\right)$.

Введем замкнутое ограниченное вьпуклое подмножество банахова пространства $C\left([0, T] ; C^{(1)}(\bar{\Omega})\right)$ :

$$
B_{1 R} \equiv\left\{v \in C\left([0, T] ; C^{(1)}(\bar{\Omega})\right):\|v\|_{1, T} \leqslant R\right\},
$$

где норма определена в (4.8). Докажем, что оператор $\mathbf{U}(u)$ действует из $B_{1 R}$ в $B_{1 R}$ и является сжимающим на $B_{1 R}$ при достаточно малом $T>0$ и достаточно большом $R>0$. Действительно,

$$
\|\mathbf{U}(u)\|_{1, T} \leqslant\left\|u_{0}\right\|_{1, T}+C T\|u\|_{1, T}^{2}+C T\|u\|_{1, T}^{3} \leqslant\left\|u_{0}\right\|_{1, T}+C T R^{2}+C T R^{3} \leqslant R
$$
при условии $\left\|u_{0}\right\|_{1, T} \leqslant R / 2, T \leqslant 2^{-1}\left(C R^{2}+C R^{3}\right)$.

Докажем теперь сжимаемость оператора $\mathbf{U}: B_{1 R} \rightarrow B_{1 R}$. Действительно,

$$
\left\|\mathbf{U}\left(u_{1}\right)-\mathbf{U}\left(u_{2}\right)\right\|_{1, T} \leqslant C R T\left\|u_{1}-u_{2}\right\|_{1, T}+C R^{2} T\left\|u_{1}-u_{2}\right\|_{1, T} \leqslant \frac{1}{2}\left\|u_{1}-u_{2}\right\|_{1, T}
$$

при условии $T \leqslant 2^{-1}\left(C R+C R^{2}\right)^{-1}$. Значит, оператор $\mathbf{U}: B_{1 R} \rightarrow B_{1 R}$ сжимаемьй.

Используя стандартный алгоритм продолжения решения интегрального уравнения (4.2) во времени, получим, что найдется такое максимальное $T_{0}^{*}$, что существует единственное решение уравнения $(4.2)$ класса $C\left([0, T] ; C^{(1)}(\bar{\Omega}) \cap C_{0}(\bar{\Omega})\right)$ для любого $T \in\left(0, T_{0}^{*}\right)$. Причем либо $T_{0}^{*}=+\infty$, либо $T_{0}^{*}<+\infty$, и тогда справедливо предельное равенство (4.1).

Тем самым, существует единственное решение интегрального уравнения (4.2) класса $C\left([0, T] ; C^{(1)}(\bar{\Omega}) \cap C_{0}(\bar{\Omega})\right)$. Из вида интегрального уравнения (4.2) легко следует, что на самом деле указанное решение принадлежит классу $C^{(1)}\left([0, T] ; C^{(1)}(\bar{\Omega}) \cap C_{0}(\bar{\Omega})\right)$.

Таким образом, мы доказали существование и единственность “ослабленного" решения задачи (1.1) в смысле определения 1.

Теорема доказана.

Заметим, что введенная ранее норма на банаховом пространстве $C\left([0, T] ; C^{(1)}(\bar{\Omega}) \cap\right.$ $\left.C_{0}(\bar{\Omega})\right)$ для выпуклых ограниченных областей с гладкой границей эквивалентна следующей:

$$
\|v\|_{T}^{\prime} \equiv \max _{(x, t) \in \bar{Q}_{T}}|\nabla v(x, t)|, \quad Q_{T} \equiv(0, T) \times \Omega .
$$

Поскольку всякое “ослабленное” решение задачи (1.1) является сильньм обобщенным, то в силу результата теоремы 2 при дополнительном условии

$$
\left\|u_{0}\right\|_{4}^{4}>\left\|\nabla u_{0}\right\|_{2}^{2}+\left\|u_{0}\right\|_{2}^{2}
$$

найдется такой момент времени $0<T_{0} \leqslant T_{2}$, что

где

$$
\lim _{t \uparrow T_{0}}\|\nabla u\|_{2}^{2}=+\infty
$$

$$
T_{2}=-\ln \left(1-\frac{\left\|\nabla u_{0}\right\|_{2}^{2}+\left\|u_{0}\right\|_{2}^{2}}{\left\|u_{0}\right\|_{4}^{4}}\right) .
$$

Но это означает, что для момента времени $T_{0}^{*}$ имеет место цепочка неравенств $0<T_{0}^{*} \leqslant$ $T_{0} \leqslant T_{2}$, т.е. за конечное время $T_{0}^{*}$ “ослабленное" решение задачи (1.1) “опрокинется":

$$
\lim _{T \uparrow T_{0}^{*}}\|u\|_{T}^{\prime}=+\infty .
$$


5. Физическая интерпретация. Приведем физическую интерпретацию полученных нами результатов. Условия теоремы 2 имеют смысл большой величины потенциальной энергии, запасенной в электрической либо магнитной среде. При этих условиях величина плотности электрических либо "магнитных" зарядов в среде со временем лавинным образом возрастает и приводит в конечном счете к пробою в рассматриваемой среде. Из результатов теоремы 3 вытекает, что за конечное время изначально и до некоторого момента времени гладкий фронт волны преврашается в ударньй, т.е. максимум производной по пространственной переменной в некоторой точке в определенньй момент времени становится равной бесконечности.

Отметим, что исходя из полученных результатов теоремы 2 для задач (1.2) и (1.3) нельзя получить как для задачи (1.1) результат об “опрокидьвании”. Что оставляет открытой задачу доказательства “опрокидывания” решений задач для уравнений (1.2) и (1.3), если, конечно, данньй результат имеет место.

\section{СПИСОК ЦИТИРОВАННОЙ ЛИТЕРАТУРЫ}

[1] Корпусов М.О., Свешников А. Г. Трехмерные нелинейные эволюционные уравнения псевдопараболического типа в задачах математической физики. II // ЖВМиМФ. 2004. Т. 44. № 11. C. 2041-2048.

[2] Benjamin T. B. Lectures on nonlinear wave motion // Lect. Appl. Math. V. 15. Providence, RI: Amer. Math. Soc., 1974. P. 3-47.

[3] Benjamin T. B., Bona J. L., Mahony J. J. Model equations for long waves in nonlinear dispersive systems // Philos. Trans. Royal Soc. London Ser. A. 1972. V. 272. № 1220. P. 47-78.

[4] Karch G. Asymptotic behavior of solutions to some pseudoparabolic equations // Math. Methods Appl. Sci. 1997. V. 20. № 3. P. 271-289.

[5] Biler P. Long time behavior of the generalized Benjamin-Bona-Mahony equation in two space dimensions // Differential Integral Equations. 1992. V. 5. № 4. P. 891-901.

[6] Goldstein J. A., Kajikiya R., Oharu S. On some nonlinear dispersive equations in several space variables // Differential Integral Equations. 1990. V. 3. № 4. P. 617-632.

[7] Zhang L. Decay of solutions of generalized BBMB equations in $n$-space dimensions // J. Nonlinear Anal. Theory Methods Appl. 1995. V. 20. P. 1343-1390.

[8] Naumkin P. I., Shishmarev I. A. Nonlinear Nonlocal Equations in the Theory of Waves. Transl. Math. Monographs. V. 133. Providence, RI: Amer. Math. Soc., 1994.

[9] Naumkin P. I. Large-time asymptotic behaviour of a step for the Benjamin-Bona-MahonyBurgers equation // Proc. Roy. Soc. Edinburgh Sect. A. 1996. V. 126. №1. P. 1-18.

[10] Avrin J., Goldstein J. A. Global existence for the Benjamin-Bona-Mahony equation in arbitrary dimensions // J. Nonlinear Anal. Theory Methods Appl. 1985. V. 9. № 8. P. 861-865.

[11] Jeffrey A., Engelbrecht J. Nonlinear dispersive waves in a relaxing medium // Wave Motion. 1980. V. 2. № 3. P. 255-266.

[12] Albert J. P. On the decay of solutions of the generalized Benjamin-Bona-Mahony equation // J. Math. Anal. Appl. 1989. V. 141. № 2. P. 527-537.

[13] Pereira J. M. Stability of multidimensional traveling waves for a Benjamin-Bona-Mahony type equation // Differential Integral Equations. 1996. V. 9. № 4. P. 849-863.

[14] Hagen T., Turi J. On a class of nonlinear BBM-like equations // Comput. Appl. Math. 1998. V. 17. № 2. P. 161-172.

[15] Medeiros L. A., Perla Menzala G. On global solutions of a nonlinear dispersive equation of Sobolev type // Bol. Soc. Brasil. Mat. 1978. V. 9. № 1. P. 49-59.

[16] Guo B., Miao Ch. On inhomogeneous GBBM equations // J. Partial Differential Equations. 1995. V. 8. № 3. P. 193-204.

[17] Mei M. $L_{q}$-decay rates of solutions for Benjamin-Bona-Mahony-Burgers equations // J. Differential Equations. 1999. V. 158. № 2. P. 314-340.

[18] Li Z. On the initial-boundary value problem for the system of multi-dimensional generalized BBM equations // Math. Appl. 1990. V. 3. № 4. P. 71-80. 
[19] Chen Yu. Remark on the global existence for the generalized Benjamin-Bona-Mahony equations in arbitrary dimension // Appl. Anal. 1988. V. 30. №1-3. P. 1-15.

[20] Liu L., Mei M. A better asymptotic profile of Rosenau-Burgers equation // J. Appl. Math. Comput. 2002. V. 131. № 1. P. 147-170.

[21] Chung Sang K., Pani Amiya K. Numerical methods for the Rosenau equation // J. Appl. Anal. 2001. V. 77. № 3-4. P. 351-369.

[22] Lee H. Y., Ohm M. R., Shin J. Y. The convergence of fully discrete Galerkin approximations of the Rosenau equation // Korean J. Comput. Appl. Math. 1999. V. 6. № 1. P. 1-13.

[23] Mei M. Long-time behavior of solution for Rosenau-Burgers equation. II // J. Appl. Anal. 1998. V. 68. № 3-4. P. 333-356.

[24] Chung S. K., Ha S. N. Finite element Galerkin solutions for the Rosenau equation // J. Appl. Anal. 1994. V. 54. № 1-2. P. 39-56.

[25] Park M. A. On the Rosenau equation in multidimensional space // J. Nonlinear Anal. Theory Methods Appl. 1993. V. 21. № 1. P. 77-85.

[26] Park M. A. Pointwise decay estimates of solutions of the generalized Rosenau equation // J. Korean Math. Soc. 1992. V. 29. № 2. P. 261-280.

[27] Корпусов М. О., Плетнер Ю. Д., Свешников А. Г. О нестационарных волнах в средах с анизотропной дисперсией // ЖКВМиМФ. 1999. Т. 39. №6. С. 968-984.

[28] Владимиров В. С. Уравнения математической физики. М.: Наука, 1988.

Московский государственный университет им. М.В. Ломоносова 